\title{
SOLUTIONS OF SOME FUNCTIONAL EQUATIONS IN A CLASS OF GENERALIZED HÖLDER FUNCTIONS
}

\author{
Maria Lupa \\ Institute of Mathematics, Częstochowa University of Technology \\ Częstochowa, Poland \\ maria.lupa@im.pcz.pl
}

Received: 17 October 2016; accepted: 15 November 2016

\begin{abstract}
The existence and uniqueness of solutions a nonlinear iterative equation in the class of $r$-times differentiable functions with the $r$-derivative satisfying a generalized Hölder condition is considered.
\end{abstract}

Keywords: iterative functional equation, generalized Hölder condition

\section{Introduction}

In $[1,2]$ the space $W_{\gamma}[a, b]\left(W_{\gamma}^{r}[a, b]\right)$ of $r$ times differentiable functions with the $r$-the derivative satisfying generalized $\gamma$-Hölder condition was introduced and some of its properties proved. In the present paper we examine the existence and uniqueness of solutions of a nonlinear iterative functional equation in this class of functions. We apply some ideas from Kuczma [3], Matkowski [4, 5] (see also Kuczma, Choczewski, Ger [6]), where differentiable solutions, Lipschitzian solutions, bounded variation solutions of different type of itrerative functional equations were investigated.

\section{Preliminaries}

Consider non-linear functional equation

$$
\varphi(x)=h(\varphi[f(x)])+g(x)
$$

where $f, g, h$ are given and $\varphi$ is a unknown function.

We accept the following notation: $I=[a, b], a, b \in R, d:=b-a, W_{\gamma}(I)-$ is the Banach space of the r-time differentiable functions defined on the interval $I$ with values in $R$, such that, for some $M \geq 0$; its $r$-th derivative satisfies the following $\gamma$-Hölder condition 


$$
\left|\varphi^{(r)}(x)-\varphi^{(r)}(\bar{x})\right| \leq M \gamma(|x-\bar{x}|), \quad \bar{x}, x \in \mathrm{I} .
$$

where a fixed function $\gamma$ satisfies the following condition (see [1, 2]):

$(\Gamma) \gamma:[0, d] \rightarrow[0, \infty)$ is increasing and concave, $\gamma(0)=0, \lim _{t \rightarrow 0^{+}} \gamma(t)=\gamma(0)$, $\lim _{t \rightarrow d^{-}} \gamma(t)=\gamma(d), \gamma_{+}^{\prime}(0)=+\infty$

We assume that

(i) $f: I \rightarrow I, f \in W_{\gamma}(I), \sup _{I}\left|\mathrm{f}^{\prime}\right| \leq 1$

(ii) $g: I \rightarrow R, g \in W_{\gamma}(I)$

(iii) $h: R \rightarrow R, h \in C^{r}, h^{(r)}$ fulfils the Lipschitz condition in $R$.

(iv) there exists $\xi \in \mathrm{I}$ such that $\lim _{n \rightarrow \infty} f^{n}(x)=\xi, x \in \mathrm{I}$, where $f^{n}$ is the $\mathrm{n}$-th iteration function $f$

(v) is analityc function at $\eta_{0}$, where $\eta_{0}$ is the solution of equation $\eta_{0}=h\left(\eta_{0}\right)+g(\xi)$

We define functions $h_{k}: I \times R^{k+1} \rightarrow R, k=0,1, \ldots, r-1$ by the formula

$$
\left\{\begin{array}{c}
h_{0}\left(x, y_{0}\right):=h\left(y_{0}\right)+g(x) \\
h_{k+1}\left(x, y_{0}, \ldots, y_{k+1}\right):=\frac{\partial h_{k}}{\partial x}+f^{\prime}(x)\left(\frac{\partial h_{k}}{\partial y_{0}} y_{1}+\cdots+\frac{\partial h_{k}}{\partial y_{k}} y_{k+1}\right) .
\end{array}\right.
$$

Lemma 1. [4]

By assumptions (i)-(iii), $h_{k}$ defined by (2) are of the form:

1. for $r=1$

2. for $r \geq 2, k=2, \ldots, r$

$$
h_{1}\left(x, y_{0}, y_{1}\right)=h^{\prime}\left(y_{0}\right) y_{1} f^{\prime}(x)+g^{\prime}(x)
$$

$$
\begin{gathered}
h_{k}\left(x, y_{0}, \ldots, y_{k}\right)=p_{k}\left(x, y_{0}, \ldots, y_{k-1}\right)+h^{\prime}\left(y_{0}\right) y_{k}\left(f^{\prime}(x)\right)^{k}+ \\
+h^{\prime}\left(y_{0}\right) y_{1} f^{(k)}(x)+g^{(k)}(x),
\end{gathered}
$$

where

$$
\begin{gathered}
p_{k}\left(x, y_{0}, \ldots, y_{k-1}\right)+h^{\prime}\left(y_{0}\right) y_{k}\left(f^{\prime}(x)\right)^{k}= \\
=\sum_{i=1}^{k} h^{(k-i+1)}\left(y_{0}\right) \sum_{\alpha_{1}+\cdots+\alpha_{i}=k-i+1} u_{\alpha_{1} \ldots \alpha_{i}, k}(x) y_{1}{ }^{\alpha_{1}} \ldots y_{i}^{\alpha_{i}}
\end{gathered}
$$

and $u_{\alpha_{1} \ldots \alpha_{i}, k}(x)$ are of the class $C^{r-k+1}$ in $I$, for all numbers $\alpha_{1}, \ldots, \alpha_{i} \in N$ such that $\alpha_{1}+\cdots+\alpha_{i}=k-i+1, k=2, \ldots, r, i=1, \ldots, k$.

\section{Remark 1.}

If (i)-(iii) are fulfilled, then $h_{r}: I \times R^{k+1} \rightarrow R$, given by

$$
\begin{gathered}
h_{r}\left(x, y_{0}, \ldots, y_{r}\right)=h^{\prime}\left(y_{0}\right) y_{1} f^{(r)}(x)+g^{(r)}(x)+ \\
+\sum_{i=1}^{r} h^{(r-i+1)}\left(y_{0}\right) \sum_{\alpha_{1}+\cdots+\alpha_{i}=r-i+1} u_{\alpha_{1} \ldots \alpha_{i}, r}(x) y_{1}^{\alpha_{1}} \ldots y_{i}^{\alpha_{i}}
\end{gathered}
$$


fulfill $\gamma$-Hölder condition for $x \in I$ and Lipschitz condition with respect to $y_{i}, i=0, \ldots, r$ in $Z:=\left[a_{0}, b_{0}\right] \times\left[a_{1}, b_{1}\right] \times \ldots \times\left[a_{r}, b_{r}\right]$. It means, that there are positive constants $m, l_{0}, \ldots, l_{r-1}$ and

$$
l_{r}=\sup _{I \times\left[a_{0}, b_{0}\right]}\left|h^{\prime}\left(f^{\prime}\right)^{r}\right|,
$$

such that for $\left(x, y_{1}, \ldots, y_{r}\right),\left(\bar{x}, \overline{y_{1}}, \ldots, \overline{y_{r}}\right) \in Z$ we have

$$
\begin{gathered}
\left|h_{r}\left(x, y_{0}, \ldots, y_{r}\right)-h_{r}\left(\bar{x}, \overline{y_{0}}, \ldots, \overline{y_{r}}\right)\right| \leq m \gamma(|x-\bar{x}|)+l_{0}\left|y_{0}-\overline{y_{0}}\right|+\cdots+ \\
+l_{r}\left|y_{r}-\overline{y_{r}}\right| .
\end{gathered}
$$

Define the functions $w_{r, i}: I \times R^{i} \rightarrow R, i=1,2, \ldots, r$ by the following formulas:

$$
w_{r, i}\left(x, y_{1}, \ldots, y_{i}\right):=\sum_{\alpha_{1}+\cdots+\alpha_{i}=r-i+1} u_{\alpha_{1} \ldots \alpha_{i}, r}(x) y_{1}^{\alpha_{1} \ldots y_{i}}{ }^{\alpha_{i}}
$$

\section{Remark 2.}

The functions $w_{r, i}$ defined by (6) fulfill $\gamma$-Hölder condition with respect to variable $\mathrm{x}$ in $I$ and Lipschitz condition with respect to the variable $y_{i}, i=1, \ldots, r$ in each set $Z_{i}:=\left[a_{1}, b_{1}\right] \times \ldots \times\left[a_{i}, b_{i}\right]$.

\section{Remark 3.}

If $f, g, h$ satisfy the assumptions (i)-(iii) and $\varphi \in W_{\gamma}(I)$ is a solution of equation (1) then the derivatives $\varphi^{(k)}, k=0, \ldots, r$ satisfy the system of equations

$$
\varphi^{(k)}(x)=h_{k}\left(x, \varphi[f(x)], \ldots, \varphi^{(k)}[f(x)]\right), \quad x \in I .
$$

If assumptions (i)-(iv) are fulfilled and $\varphi \epsilon W_{\gamma}(I)$ is a solution of equation (1) in $I$, then the numbers

$$
\eta_{k}=\varphi^{(k)}(\xi), k=0, \ldots, r
$$

satisfy the system of equations

$$
\eta_{k}=h_{k}\left(\xi, \eta_{0}, \ldots, \eta_{k}\right), k=0, \ldots, r,
$$

where $h_{k}$ are defined by (2).

\section{Remark 4.}

Let $\varphi \in W_{\gamma}(I)$ be a solution of the equation (1). Present $\varphi$ in the following form

$$
\varphi(x)=P(x)+\psi(x-\xi), x \in I=[a, b]
$$

where $\psi:[a-\xi, b-\xi] \rightarrow R$ and $P(x)=\sum_{i=0}^{r} \frac{\eta_{i}}{i !}(x-\xi)^{i}, x \in[a, b]$. 
Define the functions

$$
\begin{gathered}
\bar{f}(x):=f(x+\xi)-\xi, \quad x \in[a-\xi, b-\xi] \\
\bar{g}(x):=g(x+\xi), \quad x \in[a-\xi, b-\xi]
\end{gathered}
$$

and for $y \in R, x \in[a-\xi, b-\xi]$

$$
\bar{h}(x):=h(P[f(x+\xi)]+y)-P(x+\xi) .
$$

It follows from above definitions and equation (9) that $\psi$ satisfies the following equation

$$
\psi(x)=\bar{h}(\psi[\bar{f}(x)])+\bar{g}(x), x \in[a-\xi, b-\xi] .
$$

It is easy to prove, that if assumptions (i)-(iv) are fulfilled and $\eta_{i}, i=0, \ldots, r$, are the solution of equations (8), then the function $\varphi \in W_{\gamma}[a, b]$ satisfies the equation (1) in $[a, b]$ and the condition (7) if and only if the function $\psi$ given by (9) belongs to $W_{\gamma}[a-\xi, b-\xi]$ and satisfies

$$
\psi^{(k)}(0)=0, k=0, \ldots, r .
$$

Thus, we assume that $0 \in I$ and consider the equation (1) whose solution satisfies the condition

$$
\varphi^{(k)}(0)=0, k=0, \ldots, r .
$$

Then system of equations (8) takes the following form

$$
h_{k}(0, \ldots, 0)=0, k=0, \ldots, r .
$$

\section{Main result}

\section{Theorem 1.}

If assumptions (i)-(iii) are fulfilled, $f$ is a monotone function in the interval I, the conditions (iv) and (v) are fulfilled for $\xi=0, \eta_{0}=0$ and

$$
\begin{gathered}
h_{k}(0, \ldots, 0)=0, \quad k=1, \ldots, r \\
\left|h^{\prime}(0)\left(f^{\prime}(0)\right)^{r}\right|<1
\end{gathered}
$$

then equation (1) has exactly one solution $\varphi \in W_{\gamma}(I)$ satisfying the condition

$$
\varphi^{(k)}(0)=0, k=0, \ldots, r .
$$


Moreover, there exists a neighbourhood $U$ of the point $\xi=0$ and the number $r_{0}$ such that for a function $\varphi_{0} \in W_{\gamma}(\bar{U})$, satisfying the condition (12) and the inequality $\left\|\varphi_{0}\right\| \leq r_{0}$, a sequence of functions

$$
\varphi_{n}(x)=h\left(\varphi_{n-1}[f(x)]\right)+g(x), \quad x \in \bar{U}
$$

converges to a solution of (1) according to the norm in the space $W_{\gamma}(\bar{U})$.

Proof.

From (v) we have $h(y)=\sum_{n=0}^{\infty} a_{n} y^{n}$ in some neighbourhood of the point 0 . Denote by $R_{0}$ the radius of convergence of this series. From (11) and from the continuity of functions $\left(f^{\prime}\right)^{r}$ and $h^{\prime}$, from definition of the function $\gamma$ there exists a neighbourhood $V$ of the point $\xi=0$ and $d<R_{0}, 0<\theta<1$ such that

$$
\sup _{\bar{V} \times[-d, d]}\left|h^{\prime}\left(f^{\prime}\right)^{r}\right| \leq \theta, f(V) \subset V, \gamma(\operatorname{diam} \bar{V}) \geq \operatorname{diam} \bar{V} .
$$

From Remark 1, definition of $\gamma$ and from (13) there are positive constants $m, l_{0}, \ldots, l_{r-1}$, and $l_{r}=\theta$, that in $\bar{V} \times[-d, d]^{r+1}$ we have

$$
\begin{gathered}
\left|h_{r}\left(x, y_{0}, \ldots, y_{r}\right)-h_{r}\left(\bar{x}, \overline{y_{0}}, \ldots, \overline{y_{r}}\right)\right| \leq m \gamma(|x-\bar{x}|)+l_{0}\left|y_{0}-\overline{y_{0}}\right|+\cdots+ \\
+\theta\left|y_{r}-\overline{y_{r}}\right| .
\end{gathered}
$$

From Remark 2, definition of $\gamma$ there are in $Z_{i}=\bar{V} \times[-d, d]^{i}$ constants $B_{i, 0}, B_{i, k}$, $i=1, \ldots, r, k=1, \ldots, i$, such that

$\left|w_{r, i}\left(x, y_{1}, \ldots, y_{i}\right)-w_{r, i}\left(\bar{x}, \overline{y_{1}}, \ldots, \overline{y_{l}}\right)\right| \leq B_{i, 0} \gamma(|x-\bar{x}|)+\sum_{k=1}^{i} B_{i, k}\left|y_{k}-\overline{y_{k}}\right|$

We accept the following notation:

$$
\begin{gathered}
W_{i}:=\sup _{\bar{V} \times[-d, d]}\left|w_{r, i}\right|, \quad i=1,2, \ldots, r ; \\
H_{i}:=\sup _{\bar{V} \times[-d, d]}\left|h^{(i)}\right|, i=1,2, \ldots, r+1 ; \\
F:=\sup _{\bar{V}}\left|f^{(r)}\right| ; K \text { is a } \gamma \text {-Hölder constant of } f^{(r)} \text { in } \overline{V ;} \\
C_{\alpha_{1} \ldots \alpha_{i}, r}:=\sup _{\bar{V}}\left|u_{\alpha_{1} \ldots \alpha_{i}, r}\right|, i=1,2, \ldots, r, \alpha_{1}+\cdots+\alpha_{i}=r-i+1 ; \\
D_{\alpha_{1} \ldots \alpha_{i}, r}:=\sup _{\bar{V}}\left|u_{\alpha_{1} \ldots \alpha_{i}, r}^{\prime}\right|, i=1,2, \ldots, r, \alpha_{1}+\cdots+\alpha_{i}=r-i+1 .
\end{gathered}
$$


By $\sum a_{\alpha_{1} \ldots \alpha_{i}, r}$ we denote the sum of $a_{\alpha_{1} \ldots \alpha_{i}, r}$ for all $\alpha_{1}, \ldots, \alpha_{i} \in N$ such that $\alpha_{1}+\cdots+\alpha_{i}=r-i+1, i=1,2, \ldots, r$.

In view of Lemma 1 , we have

$$
u_{0 \ldots 01_{i}, r}=\left(f^{\prime}\right)^{r}
$$

and, from (13), we get

$$
\left|h^{\prime}(y) u_{0 \ldots 0 i_{i}, r}(x)\right| \leq \theta, x \in \bar{V}, y \epsilon[-d, d]
$$

Let us take $c_{1} \epsilon(0, b-a], c_{1} \leq \gamma\left(c_{1}\right) \leq 1$ and

$$
\gamma\left(c_{1}\right) \sum_{i=0}^{r-1} l_{i} c_{1}^{r-i-1}<1-\theta
$$

Put

$$
r_{0}:=\frac{m}{1-\theta-\gamma\left(c_{1}\right) \sum_{i=0}^{r-1} l_{i} c_{1}^{r-i-1}}
$$

Then let's take $c_{2} \epsilon(0, b-a]$ such that $c_{2} \leq \gamma\left(c_{2}\right) \leq \min \left\{1, \frac{d}{r_{0}}\right\}$ and

$$
\begin{gathered}
l_{0}:=H_{1} F\left(\gamma\left(c_{2}\right)\right)^{r-1}+H_{2} F\left(\gamma\left(c_{2}\right)\right)^{2 r}+H_{1} K\left(\gamma\left(c_{2}\right)\right)^{r}+H_{2} F r_{0}\left(\gamma\left(c_{2}\right)\right)^{2 r}+ \\
+H_{2} K r_{0}\left(\gamma\left(c_{2}\right)\right)^{2 r+1}+F r_{0}\left(\gamma\left(c_{2}\right)\right)^{2 r} \sum_{n=2}^{\infty} n(n-1)^{2}\left|a_{n}\right| r_{0}^{n-2}\left(\gamma\left(c_{2}\right)\right)^{(n-2)(r+1)} \\
+\left(\gamma\left(c_{2}\right)\right)^{r} \sum_{i=1}^{r} W_{i} \sum_{n=r-i+2}^{\infty}\left|a_{n}\right| n(n-1)(n-r+i-2)^{2} r_{0}^{n-r+i-2} \\
\cdot\left(\gamma\left(c_{2}\right)\right)^{(n-r+i-2)(r+1)}+ \\
+\left(\gamma\left(c_{2}\right)\right)^{r+1}\left(\sum_{i=1}^{r} H_{r-i+2}\left(B_{i, 0}+2 r_{0} \sum_{i=1}^{i} B_{i, k}\left(\gamma\left(c_{2}\right)\right)^{r-k}\right)\right)+ \\
+\sum_{i=1}^{r-1} H_{r-i+1} \sum C_{\alpha_{1} \ldots \alpha_{i}, r} r_{0}^{r-i}\left(\gamma\left(c_{2}\right)\right)^{r \alpha_{1}+\cdots+(r-i+1) \alpha_{i}-1}(r-i+1)^{2}+ \\
+H_{1} \sum C_{\alpha_{1} \ldots \alpha_{r-1} 0, r}\left(\gamma\left(c_{2}\right)\right)^{r \alpha_{1}+(r-1) \alpha_{2}+\cdots+2 \alpha_{r-1}-1}+ \\
+\sum_{i=1}^{r} H_{r-i+1} r_{0}^{r-i}(r-i+1) \sum D_{\alpha_{1} \ldots \alpha_{i}, r}\left(\gamma\left(c_{2}\right)\right)^{r \alpha_{1}+\cdots+(r-i+1) \alpha_{i}}<1-\theta . \quad \text { (23) }
\end{gathered}
$$

Choose $c \leq \min \left\{c_{1}, c_{2}\right\}$. Of course $c \leq \gamma(c) \leq \frac{d}{r_{0}}$. We will select a neighborhood of zero $U \subset V$ such that $f(U) \subset U$ and $\operatorname{diam} \bar{U} \leq c$.

Consider the Banach space $W_{\gamma}(\bar{U})$ with the norm: 


$$
\|\varphi\|:=\sum_{k=0}^{r}\left|\varphi^{(k)}(0)\right|+\sup \left\{\frac{\left|\varphi^{(r)}(x)-\varphi^{(r)}(\bar{x})\right|}{\gamma(|x-\bar{x}|)} ; x, \bar{x} \in \bar{U}, x \neq \bar{x}\right\} .
$$

Let us define the set

$$
A_{r_{0}}:=\left\{\varphi \in W_{\gamma}(\bar{U}), \varphi^{(k)}(0)=0, k=0, \ldots, r,\|\varphi\| \leq r_{0}\right\} .
$$

Note that $A_{r_{0}}$ is a closed subset of Banach space $W_{\gamma}(\bar{U})$ and for $\varphi \in A_{r_{0}}$ the norm is expressed by the formula

$$
\|\varphi\|:=\sup \left\{\frac{\left|\varphi^{(r)}(x)-\varphi^{(r)}(\bar{x})\right|}{\gamma(|x-\bar{x}|)} ; x, \bar{x} \in \bar{U}, x \neq \bar{x}\right\}
$$

Thus, the set $A_{r_{0}}$ with the metric $\varrho\left(\varphi_{1}, \varphi_{2}\right):=\left\|\varphi_{1}-\varphi_{2}\right\|$ is a complete metric space.

By the mean value theorem and by definition of the number of $\mathrm{c}$ we have for $\varphi \epsilon A_{r_{0}}$

$$
\sup \left|\varphi^{(k)}\right| \leq c^{r-k} \gamma(c) r_{0} \leq \gamma(c) r_{0} \leq d, k=0, \ldots, r
$$

and so $\varphi^{(k)} \epsilon[-d, d], k=0, \ldots, r$.

For $\varphi \epsilon A_{r_{0}}$ define the transformation $T$ by the formula

$$
(T \varphi)(x):=h(\varphi[f(x)])+g(x), \quad x \in \bar{U} .
$$

We will show that $T\left(A_{r_{0}}\right) \subset A_{r_{0}}$.

Based on Remarks 1 and 3 the function $\psi:=T \varphi$ belongs to $W_{\gamma}(\bar{U})$, from (iv) and (10), (12) appears that $\psi^{(k)}(0)=0, k=0, \ldots, r$. Then using the formulas (12), (13), (22), (25) and the assumption (i) we obtain

$$
\begin{gathered}
\left|\psi^{(r)}(x)-\psi^{(r)}(\bar{x})\right| \leq m \gamma(|x-\bar{x}|)+l_{0}|\varphi[f(x)]-\varphi[f(\bar{x})]|+\cdots+ \\
+l_{r-1}\left|\varphi^{(r-1)}[f(x)]-\varphi^{(r-1)}[f(\bar{x})]\right|+\theta\left|\varphi^{(r)}[f(x)]-\varphi^{(r)}[f(\bar{x})]\right| \leq \\
\left(m+l_{0} c^{r-1} \gamma(c) r_{0}+\cdots+l_{r-1} \gamma(c) r_{0}+\theta r_{0}\right) \gamma(|x-\bar{x}|) \leq r_{0} \gamma(|x-\bar{x}|) .
\end{gathered}
$$

Which means from (24) that $\|T \varphi\| \leq r_{0}$. Thus $T\left(A_{r_{0}}\right) \subset A_{r_{0}}$.

Now we prove that $T$ is a contraction map. Let us put $\psi_{1}:=T \varphi_{1}, \psi_{2}:=T \varphi_{2}$. Basing on formulas (4)-(5) of Lemma 1 and from (24) we have 


$$
\begin{aligned}
& \left|\psi_{1}^{(r)}(x)-\psi_{1}^{(r)}(\bar{x})-\psi_{2}^{(r)}(x)+\psi_{2}^{(r)}(\bar{x})\right|= \\
& =\mid h^{\prime}\left(\varphi_{1}[f(x)]\right) \varphi_{1}^{\prime}[f(x)] f^{(r)}(x)-h^{\prime}\left(\varphi_{1}[f(\bar{x})]\right) \varphi_{1}^{\prime}[f(\bar{x})] f^{(r)}(\bar{x})+ \\
& -h^{\prime}\left(\varphi_{2}[f(x)]\right) \varphi_{2}^{\prime}[f(x)] f^{(r)}(x)+h^{\prime}\left(\varphi_{2}[f(\bar{x})]\right) \varphi_{2}^{\prime}[f(\bar{x})] f^{(r)}(\bar{x})+ \\
& +\sum_{i=1}^{r}\left(h^{(r-i+1)}\left(\varphi_{1}[f(x)]\right) w_{r, i}\left(x, \varphi_{1}^{\prime}[f(x)], \ldots, \varphi_{1}^{(i)}[f(x)]\right)+\right. \\
& -h^{(r-i+1)}\left(\varphi_{1}[f(\bar{x})]\right) w_{r, i}\left(\bar{x}, \varphi_{1}^{\prime}[f(\bar{x})], \ldots, \varphi_{1}^{(i)}[f(\bar{x})]\right)+ \\
& -h^{(r-i+1)}\left(\varphi_{2}[f(x)]\right) w_{r, i}\left(x, \varphi_{2}^{\prime}[f(x)], \ldots, \varphi_{2}^{(i)}[f(x)]\right)+ \\
& \left.+h^{(r-i+1)}\left(\varphi_{2}[f(\bar{x})]\right) w_{r, i}\left(\bar{x}, \varphi_{2}^{\prime}[f(\bar{x})], \ldots, \varphi_{2}^{(i)}[f(\bar{x})]\right)\right) \mid \leq \\
& \left|h^{\prime}\left(\varphi_{1}[f(x)]\right)\right|\left|f^{(r)}(x)\right|\left|\varphi_{1}^{\prime}[f(x)]-\varphi_{1}^{\prime}[f(\bar{x})]-\varphi_{2}^{\prime}[f(x)]+\varphi_{2}^{\prime}[f(\bar{x})]\right|+ \\
& +\left|f^{(r)}(x)\right|\left|\varphi_{1}^{\prime}[f(\bar{x})]-\varphi_{2}^{\prime}[f(\bar{x})]\right|\left|h^{\prime}\left(\varphi_{1}[f(x)]\right)-h^{\prime}\left(\varphi_{1}[f(\bar{x})]\right)\right|+ \\
& +\left|h^{\prime}\left(\varphi_{1}[f(\bar{x})]\right)\right|\left|\varphi_{1}^{\prime}[f(\bar{x})]-\varphi_{2}^{\prime}[f(\bar{x})]\right|\left|f^{(r)}(x)-f^{(r)}(\bar{x})\right|+ \\
& +\left|f^{(r)}(x)\right|\left|\varphi_{2}^{\prime}[f(x)]-\varphi_{2}^{\prime}[f(\bar{x})]\right|\left|h^{\prime}\left(\varphi_{1}[f(x)]\right)-h^{\prime}\left(\varphi_{2}[f(x)]\right)\right|+ \\
& +\left|\varphi_{2}[f(\bar{x})]\right|\left|f^{(r)}(x)-f^{(r)}(\bar{x})\right|\left|h^{\prime}\left(\varphi_{1}[f(x)]\right)-h^{\prime}\left(\varphi_{2}[f(x)]\right)\right|+ \\
& +\left|\varphi_{2}[f(\bar{x})]\right|\left|f^{(r)}(\bar{x})\right|\left|h^{\prime}\left(\varphi_{1}[f(x)]\right)-h^{\prime}\left(\varphi_{2}[f(x)]\right)-h^{\prime}\left(\varphi_{1}[f(\bar{x})]\right)+h^{\prime}\left(\varphi_{2}[f(\bar{x})]\right)\right|+ \\
& +\sum_{i=1}^{r}\left(\left|w_{r, i}\left(x, \varphi_{1}^{\prime}[f(x)], \ldots, \varphi_{1}^{(i)}[f(x)]\right)\right| \mid h^{(r-i+1)}\left(\varphi_{1}[f(x)]\right)-h^{(r-i+1)}\left(\varphi_{1}[f(\bar{x})]\right)+\right. \\
& -h^{(r-i+1)}\left(\varphi_{2}[f(x)]\right)+h^{(r-i+1)}\left(\varphi_{2}[f(\bar{x})]\right) \mid+ \\
& +\left|h^{(r-i+1)}\left(\varphi_{1}[f(\bar{x})]\right)-h^{(r-i+1)}\left(\varphi_{2}[f(\bar{x})]\right)\right| . \\
& \cdot\left|w_{r, i}\left(x, \varphi_{1}^{\prime}[f(x)], \ldots, \varphi_{1}^{(i)}[f(x)]\right)-w_{r, i}\left(\bar{x}, \varphi_{1}^{\prime}[f(\bar{x})], \ldots, \varphi_{1}^{(i)}[f(\bar{x})]\right)\right|+ \\
& +\left|h^{(r-i+1)}\left(\varphi_{2}[f(x)]\right)-h^{(r-i+1)}\left(\varphi_{2}[f(\bar{x})]\right)\right| . \\
& \cdot\left|w_{r, i}\left(x, \varphi_{1}^{\prime}[f(x)], \ldots, \varphi_{1}^{(i)}[f(x)]\right)-w_{r, i}\left(x, \varphi_{2}^{\prime}[f(x)], \ldots, \varphi_{2}^{(i)}[f(x)]\right)\right|+ \\
& +\left|h^{(r-i+1)}\left(\varphi_{2}[f(\bar{x})]\right)\right| \mid w_{r, i}\left(x, \varphi_{1}^{\prime}[f(x)], \ldots, \varphi_{1}^{(i)}[f(x)]\right)-w_{r, i}\left(\bar{x}, \varphi_{1}^{\prime}[f(\bar{x})], \ldots, \varphi_{1}^{(i)}[f(\bar{x})]\right)+ \\
& -w_{r, i}\left(x, \varphi_{2}^{\prime}[f(x)], \ldots, \varphi_{2}^{(i)}[f(x)]\right)+w_{r, i}\left(x, \varphi_{2}^{\prime}[f(\bar{x})], \ldots, \varphi_{2}^{(i)}[f(\bar{x})]\right) \mid \text {. }
\end{aligned}
$$

Note, that if $\varphi_{1}, \varphi_{2} \in A_{r_{0}}$, then in view of the mean value theorem, from the definition of the number $c$ and from (i) we have the following inequalities

$$
\sup _{\bar{U}}\left|\varphi_{i}^{(k)}\right| \leq r_{0} c^{r-k} \gamma(c) \leq r_{0}(\gamma(c))^{r-k+1}, \quad k=0, \ldots, r, \quad i=1,2 ;
$$




$$
\begin{aligned}
& \left|\varphi_{1}^{(k)}[f(x)]-\varphi_{1}^{(k)}[f(\bar{x})]\right| \leq r_{0}(\gamma(c))^{r-k} \gamma(|x-\bar{x}|), \quad k=0, \ldots, r, \quad x, \bar{x} \in \bar{U} ; \\
& \left|\varphi_{1}^{(k)}[f(x)]-\varphi_{2}^{(k)}[f(x)]\right| \leq\left\|\varphi_{1}-\varphi_{2}\right\|(\gamma(c))^{r-k+1}, \quad k=0, \ldots, r, \quad x \in \bar{U} ; \\
& \left|\varphi_{1}^{(k)}[f(x)]-\varphi_{1}^{(k)}[f(\bar{x})]-\varphi_{2}^{(k)}[f(x)]+\varphi_{2}^{(k)}[f(\bar{x})]\right| \leq\left\|\varphi_{1}-\varphi_{2}\right\|(\gamma(c))^{r-k} \gamma(|x-\bar{x}|) \\
& k=0, \ldots, r, \quad x, \bar{x} \in \bar{U}
\end{aligned}
$$

By induction on $l \in N$ we also obtain:

$$
\begin{gathered}
\left|\left(\varphi_{1}^{(k)}[f(x)]\right)^{l}-\left(\varphi_{1}^{(k)}[f(\bar{x})]\right)^{l}-\left(\varphi_{2}^{(k)}[f(x)]\right)^{l}+\left(\varphi_{2}^{(k)}[f(\bar{x})]\right)^{l}\right| \leq \\
l^{2} r_{0}^{l-1}(\gamma(c))^{l(r-k)+l-1}\left\|\varphi_{1}-\varphi_{2}\right\| \gamma(|x-\bar{x}|), \quad k=0, \ldots, r, \quad x, \bar{x} \in \bar{U}, \quad l=1,2, \ldots
\end{gathered}
$$

From (v) and by selection of $d$ we have uniform and absolute convergence of the series

$$
h^{\prime}(y)=\sum_{n=1}^{\infty} n a_{n} y^{n-1} \text { for } y \in[-d, d]
$$

Let's consider the expression:

$$
\begin{gathered}
\left|h^{\prime}\left(\varphi_{1}[f(x)]\right)-h^{\prime}\left(\varphi_{1}[f(\bar{x})]\right)-h^{\prime}\left(\varphi_{2}[f(x)]\right)+h^{\prime}\left(\varphi_{2}[f(\bar{x})]\right)\right|= \\
=\left|\sum_{n=2}^{\infty} n a_{n}\left(\left(\varphi_{1}[f(x)]\right)^{n-1}-\left(\varphi_{1}[f(\bar{x})]\right)^{n-1}-\left(\varphi_{2}[f(x)]\right)^{n-1}+\left(\varphi_{2}[f(\bar{x})]\right)^{n-1}\right)\right| .
\end{gathered}
$$

From (30) we obtain

$$
\begin{gathered}
\quad\left|\left(\varphi_{1}[f(x)]\right)^{n-1}-\left(\varphi_{1}[f(\bar{x})]\right)^{n-1}-\left(\varphi_{2}[f(x)]\right)^{n-1}+\left(\varphi_{2}[f(\bar{x})]\right)^{n-1}\right| \leq \\
\leq(n-1)^{2} r_{0}^{n-2}(\gamma(c))^{(n-1) r+n-2}\left\|\varphi_{1}-\varphi_{2}\right\| \gamma(|x-\bar{x}|), \quad x, \bar{x} \in \bar{U}, \quad n=2,3, \ldots .
\end{gathered}
$$

Note that a series

$$
\sum_{n=2}^{\infty} A_{n} \text { where } A_{n}:=n\left|a_{n}\right|(n-1)^{2} r_{0}^{n-2}(\gamma(c))^{(n-1) r+n-2}
$$

converges, because the numbers $c, d$ have been selected in such a way that

$$
\lim _{n \rightarrow \infty}\left|\frac{A_{n+1}}{A_{n}}\right|=\frac{r_{0}}{R_{0}}(\gamma(c))^{r+1} \leq \frac{r_{0} \gamma(c)}{R_{0}} \leq \frac{d}{R_{0}}<1 .
$$




\section{Therefore}

$$
\begin{aligned}
& \left|h^{\prime}\left(\varphi_{1}[f(x)]\right)-h^{\prime}\left(\varphi_{1}[f(\bar{x})]\right)-h^{\prime}\left(\varphi_{2}[f(x)]\right)+h^{\prime}\left(\varphi_{2}[f(\bar{x})]\right)\right| \leq \\
\leq & \sum_{n=2}^{\infty} n(n-1)^{2}\left|a_{n}\right| r_{0}^{n-2}(\gamma(c))^{(n-1) r+n-2}\left\|\varphi_{1}-\varphi_{2}\right\| \gamma(|x-\bar{x}|), \quad x, \bar{x} \in \bar{U} .
\end{aligned}
$$

Similarly for $x, \bar{x} \in \bar{U}, i=1, \ldots, r$ we get

$$
\begin{gathered}
\left|h^{(r-i+1)}\left(\varphi_{1}[f(x)]\right)-h^{(r-i+1)}\left(\varphi_{1}[f(\bar{x})]\right)-h^{(r-i+1)}\left(\varphi_{2}[f(x)]\right)+h^{(r-i+1)}\left(\varphi_{2}[f(\bar{x})]\right)\right| \leq \\
\sum_{n=r-i+2}^{\infty}\left|a_{n}\right| n \ldots(n-r+i)(n-r+i-1)^{2} r_{0}^{n-r+i-2}(\gamma(c))^{(n-r+i-1) r+n-r+i-2}\left\|\varphi_{1}-\varphi_{2}\right\| \gamma(|x-\bar{x}|) .
\end{gathered}
$$

By induction and from (26)-(29) we have

$$
\begin{gathered}
\left|\left(\varphi_{1}^{\prime}[f(x)]\right)^{\alpha_{1}} \ldots\left(\varphi_{1}^{(i)}[f(\bar{x})]\right)^{\alpha_{i}}-\left(\varphi_{2}^{\prime}[f(x)]\right)^{\alpha_{1}} \ldots\left(\varphi_{2}^{(i)}[f(\bar{x})]\right)^{\alpha_{i}}\right| \leq \\
\leq\left(\alpha_{1}+\ldots+\alpha_{i}\right) r_{0}^{\alpha_{1}+\ldots+\alpha_{i}-1}(\gamma(c))^{r \alpha_{1}+\ldots+(r-i+1) \alpha_{i}}\left\|\varphi_{1}-\varphi_{2}\right\|, \\
\alpha_{1}, \ldots, \alpha_{i} \in N, i=1, \ldots, r, \quad x, \bar{x} \in \bar{U}, \varphi_{1}, \varphi_{2} \in A_{r_{0}} \\
\left|\left(\varphi_{2}^{\prime}[f(x)]\right)^{\alpha_{1}} \ldots\left(\varphi_{2}^{(i)}[f(x)]\right)^{\alpha_{i}}-\left(\varphi_{2}^{\prime}[f(\bar{x})]\right)^{\alpha_{1}} \ldots\left(\varphi_{2}^{(i)}[f(\bar{x})]\right)^{\alpha_{i}}\right| \leq \\
\leq\left(\alpha_{1}+\ldots+\alpha_{i}\right) r_{0}^{\alpha_{1}+\ldots+\alpha_{i}-1}(\gamma(c))^{r \alpha_{1}+\ldots+(r-i+1) \alpha_{i}-1} \gamma(|x-\bar{x}|), \\
i=1, \ldots, r, \quad x, \bar{x} \in \bar{U}, \quad \varphi_{2} \in A_{r_{0}} .
\end{gathered}
$$

Now from (33) and (34) we get

$$
\begin{gathered}
\mid\left(\varphi_{1}^{\prime}[f(x)]\right)^{\alpha_{1}} \ldots\left(\varphi_{1}^{(i)}[f(x)]\right)^{\alpha_{i}}-\left(\varphi_{1}^{\prime}[f(\bar{x})]\right)^{\alpha_{1}} \ldots\left(\varphi_{1}^{(i)}[f(\bar{x})]\right)^{\alpha_{i}}+ \\
-\left(\varphi_{2}^{\prime}[f(x)]\right)^{\alpha_{1}} \ldots\left(\varphi_{2}^{(i)}[f(x)]\right)^{\alpha_{i}}+\left(\varphi_{2}^{\prime}[f(\bar{x})]\right)^{\alpha_{1}} \ldots\left(\varphi_{2}^{(i)}[f(\bar{x})]\right)^{\alpha_{i}} \mid \leq \\
\leq\left(\alpha_{1}+\ldots+\alpha_{i}\right)^{2} r_{0}^{\alpha_{1}+\ldots+\alpha_{i}-1}(\gamma(c))^{r \alpha_{1}+\ldots+(r-i+1) \alpha_{i}-1}\left\|\varphi_{1}-\varphi_{2}\right\| \gamma(|x-\bar{x}|), \\
i=1, \ldots, r, \quad x, \bar{x} \in \bar{U}, \varphi_{1}, \varphi_{2} \in A_{r_{0}} .
\end{gathered}
$$

From (6), by the mean value theorem and from (33) and (34) we get 


$$
\begin{gathered}
\mid w_{r, i}\left(x, \varphi_{1}^{\prime}[f(x)], \ldots, \varphi_{1}^{(i)}[f(x)]\right)-w_{r, i}\left(\bar{x}, \varphi_{1}^{\prime}[f(\bar{x})], \ldots, \varphi_{1}^{(i)}[f(\bar{x})]\right)+ \\
-w_{r, i}\left(x, \varphi_{2}^{\prime}[f(x)], \ldots, \varphi_{2}^{(i)}[f(x)]\right)+w_{r, i}\left(\bar{x}, \varphi_{2}^{\prime}[f(\bar{x})], \ldots, \varphi_{2}^{(i)}[f(\bar{x})] \mid \leq\right. \\
\leq \sum\left|u_{\alpha_{1} \ldots \alpha_{i}, r}(x)\right|(r-i+1)^{2} r_{0}^{r-i}(\gamma(c))^{r \alpha_{1}+\ldots+(r-i+1) \alpha_{i}-1}\left\|\varphi_{1}-\varphi_{2}\right\| \gamma(|x-\bar{x}|)+ \\
+\sum\left|u_{\alpha_{1} \ldots \alpha_{i}, r}(z)\right|(r-i+1) r_{0}^{r-i}(\gamma(c))^{r \alpha_{1}+\ldots+(r-i+1) \alpha_{i}}\left\|\varphi_{1}-\varphi_{2}\right\| \gamma(|x-\bar{x}|) \\
i=1,2, \ldots, r, x, \bar{x} \in \bar{U}, z \text { is between } x \text { and } \bar{x} .
\end{gathered}
$$

Now, from (15)-(22), (27)-(32) and (36) we get

$$
\begin{gathered}
\left|\psi_{1}^{(r)}(x)-\psi_{1}^{(r)}(\bar{x})-\psi_{2}^{(r)}(x)+\psi_{2}^{(r)}(\bar{x})\right| \leq \\
\leq\left(H_{1} F(\gamma(c))\right)^{r-1}+H_{2} F r_{0}(\gamma(c))^{2 r}+H_{1} K(\gamma(c))^{r}+ \\
+H_{2} F r_{0}(\gamma(c))^{2 r}+H_{2} K r_{0}(\gamma(c))^{2 r+1}+ \\
+F r_{0}(\gamma(c))^{2 r} \sum_{n=2}^{\infty}\left|a_{n}\right| n(n-1)^{2} r_{0}^{n-2}(\gamma(c))^{(n-2)(r+1)}+ \\
+\sum_{i=1}^{r} W_{i}(\gamma(c))^{r} \sum_{n=r-i+2}^{\infty}\left|a_{n}\right| n(n-1) \ldots(n-r+i)(n-r+i-1)^{2} r_{0}^{n-r+i-2} . \\
\cdot(\gamma(c))^{(n-r+i-2)(r-1) r}+(\gamma(c))^{r+1}\left(\sum_{i=1}^{r} H_{r-i+2}\left(B_{i, 0}+2 r_{0} \sum_{k=1}^{i} B_{i, k}(\gamma(c))^{r-k}\right)\right)+ \\
+\sum_{i=1}^{r-1} H_{r-i+1} \sum_{\alpha_{1} \ldots \alpha_{i}, r}(r-i+1)^{2} r_{0}^{r-i}(\gamma(c))^{r \alpha_{1}+\ldots+(r-i+1) \alpha_{i}-1}+ \\
+H_{1} \sum_{\alpha_{1}+\ldots+\alpha_{r-1}=1, \alpha_{r}=0} C_{\alpha_{1} \ldots \alpha_{r-1} 0, r}(\gamma(c))^{r \alpha_{1}+\ldots+2 \alpha_{r-1}-1}+ \\
+\sum_{i=1}^{r-1} H_{r-i+1} \sum_{\alpha_{1} \ldots \alpha_{i}, r}(r-i+1) r_{0}^{r-i}(\gamma(c))^{r \alpha_{1}+\ldots+(r-i+1) \alpha_{i}}+ \\
\left.+\operatorname{Sup}_{\bar{U}}\left|h^{\prime} u_{0, \ldots 1, r}\right|\right)\left\|\varphi_{1}-\varphi_{2}\right\| \gamma(|x-\bar{x}|) \leq\left(l_{0}+\theta\right)\left\|\varphi_{1}-\varphi_{2}\right\| \gamma(|x-\bar{x}|) .
\end{gathered}
$$

Putting $L=l_{0}+\theta$ and making use of definition (24) of the norm in $W_{\gamma}(\bar{U})$ we have

$$
\left\|\psi_{1}-\psi_{2}\right\| \leq L\left\|\varphi_{1}-\varphi_{2}\right\|
$$

which means that $\rho\left(\psi_{1}, \psi_{2}\right) \leq L \rho\left(\varphi_{1}, \varphi_{2}\right)$, where $L<1$ in view on (23). 
By the Banach fixed point theorem, there is exactly one solution $\bar{\varphi} \in W_{\gamma}(\bar{U})$ of (1) satisfying the condition (12). This solution is given as the limit of series of successive approximations.

$$
\varphi_{n}(x)=h\left(\varphi_{n-1}[f(x)]\right)+g(x), \quad n \in N, \quad x \in \bar{U}
$$

where $\varphi_{0} \in A_{r_{0}}$. This sequence converges in the sense of the norm of $W_{\gamma}(\bar{U})$. By Lemma 4 in [7], there exists the unique extension $\varphi$ of $\bar{\varphi}$ to the whole interval $I$ such that $\varphi=\bar{\varphi}$ for $x \in \bar{U}$ and $\varphi$ satisfies the equation (1) in $I$. This completes the proof.

\section{Conclusions}

In this paper, applying the Banach contraction principle, a theorem on the existence and uniqueness of $W_{\gamma}$-solutions of nonlinear iterative functional equation (1) has been proved. The suitable unique solution is determined as a limit of sequence of successive approximations.

\section{References}

[1] Lupa M., A special case of generalized Hölder functions, Journal of Applied Mathematics and Computational Mechanics 2014, 13(4), 81-89.

[2] Lupa M., On a certain property of generalized Hölder functions, Journal of Applied Mathematics and Computational Mechanics 2015, 14(4), 127-132.

[3] Kuczma M., Functional Equations in a Single Variable, PWN, Warszawa 1968.

[4] Matkowski J., On the uniqueness of differentiable solutions of a functional equation, Bulletin de l'Academie des Sciences, Serie des sciences math., astr. et phys. 1970, XVIII, 5, 253-255.

[5] Matkowski J., On the existence of differentiable solutions of a functional equation, Bulletin de l'Academie des Sciences, Serie des sciences math., astr. et phys. 1971, XIX, 1, 19-21.

[6] Kuczma M., Choczewski B., Ger R., Iterative Functional Equations, Cambridge University Press, Cambridge-New York-Port Chester-Melbourne-Sydney 1990.

[7] Lupa M., On solutions of a functional equation in a special class of functions, Demonstratio Mathematica 1993, XXVI, 1, 137-147. 